\title{
Sensitivity-Based Interval PDE Observer for Battery SOC Estimation
}

\author{
H. E. Perez and S. J. Moura
}

\begin{abstract}
Complex multi-partial differential equation (PDE) electrochemical battery models are characterized by parameters that are often difficult to measure or identify. This parametric uncertainty influences the state estimates of electrochemical model-based observers for applications such as state-of-charge (SOC) estimation. This paper develops a sensitivity-based interval observer that maps bounded parameter uncertainty to state estimation intervals, within the context of electrochemical PDE models and SOC estimation. Theoretically, this paper extends the notion of interval observers to PDE models using a sensitivity-based approach. Practically, this paper quantifies the sensitivity of battery state estimates to parameter variations, enabling robust battery management schemes.
\end{abstract}

\section{INTRODUCTION}

This paper develops a sensitivity-based interval partial differential equation (PDE) observer for state-of-charge (SOC) estimation in batteries, using an electrochemical-based model with bounded uncertain parameters. The goal is to generate an interval estimate of battery SOC that mathematically relates parametric uncertainty to estimation uncertainty.

Batteries are ubiquitous in applications ranging from smart phones to electrified transportation. In telecommunications, there were 5.2B active mobile handsets and over 1.7B mobile phone sales worldwide for 2012 [1]. In electrified transportation, there were 53,000 plug-in electric vehicles sold in the U.S. for 2012 [2]. Despite growing sales, "range anxiety" is considered the largest inhibitor of electrified transportation. These facts provide overwhelming motivation for accurate and robust SOC estimation to maximize battery performance and lifetime.

To this end, electrochemical models [3] have attracted significant attention from battery controls researchers, due to their potential for high accuracy predictions. The parameters of these models, however, are often characterized by a bounded interval of uncertainty. In this paper, we seek to generate interval state estimates of lithium-ion concentration, given a simple PDE electrochemical model, measurements of current and voltage, and bounds on parameter values. Mathematically, we abstract this problem as an interval PDE observer design task, based upon sensitivity equations. The two relevant bodies of literature include electrochemical model-based SOC estimation and interval observers.

Electrochemical battery models capture the spatiotemporal dynamics of lithium ion concentration, electrode potential, and Butler-Volmer kinetics. Most models in the battery controls literature have been derived from the DoyleFuller-Newman model [4], which includes PDEs, ODEs in

H. E. Perez and S. J. Moura are with Civil and Environmental Engineering, University of California, Berkeley, CA 94720, USA E-mail: $\{$ heperez, smoura\} @berkeley.edu space, ODEs in time, and nonlinear algebraic equations. This system of equations is generally too complex for state observer design. Consequently, researchers have combined various model reduction and estimation techniques to perform SOC estimation. These include residue grouping/Kalman filters [5], electrode averaging/extended Kalman filters [6], output error injection [7], and adaptive PDE observers [8] The underlying electrochemical models, however, are characterized by parametric uncertainty [9]. The sensitivity of state estimates to parametric uncertainty has not been addressed in the literature.

Parallel to battery SOC estimation is the theory of interval observers. In state estimation and filtering schemes, process and measurement noises are often assumed to be Gaussian. In contrast, interval or bounding observers assume process and measurement disturbances are unknown but bounded [10], [11]. We develop a similar yet different approach that assumes specified parameters are unknown but bounded. The approach is based upon sensitivity analysis [12] and developed within the context of PDE state observers [13].

This paper connects the aforementioned bodies of literature by developing a novel interval observer based upon sensitivity equations. As such, the results provide two key contributions. First, a sensitivity-based interval observer is proposed that relates parametric uncertainty to an interval state estimate, for battery SOC estimation. Second, the sensitivity equations provide a metric for ranking parameter sensitivity, as illustrated in Section VI. We develop these results within the context of an electrochemical PDE model of lithium-ion batteries.

This paper is organized as follows: The single particle model and corresponding backstepping observer have been designed in [8], [14], but are provided in Sections II and III, respectively, for completeness. Sections IV and V develop the observer sensitivity equations and formulate the interval observer. Finally, Sections VI and VII provide simulation results and a summary of the key contributions.

\section{Electrochemical Model Development}

The sensitivity-based interval PDE observer is based upon an observer-oriented electrochemical model and backstepping observer designed in [8], [14]. For completeness and context, we summarize the model development here.

\section{A. Single Particle Model}

We consider the simplest of electrochemical battery models, known as the "single particle model" (SPM) [15]. This model is derived from the complete Doyle-Fuller-Newman model [4] by assuming the electrolyte Li concentration is 


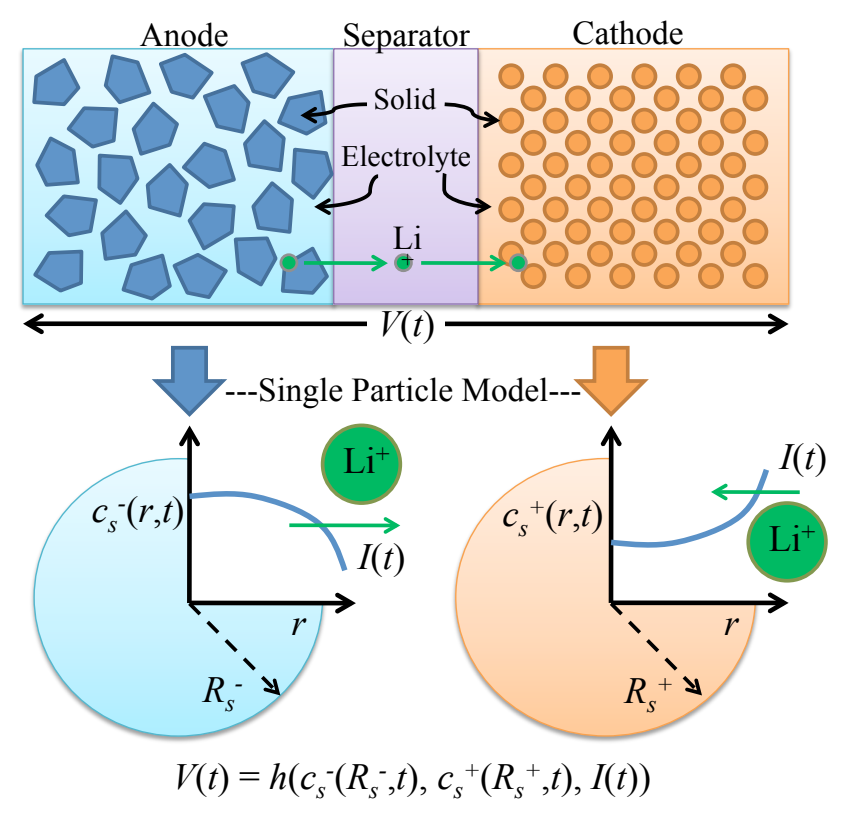

Fig. 1. Each electrode is idealized as a single porous spherical particle. This model results from assuming the electrolyte concentration is constant in space and time [3]

constant in space and time [3]. This approximation is reasonably valid for low C-rates (i.e. low current magnitudes).

As shown in Fig. 1 the model consists of two diffusion PDEs in spherical coordinates governing Li concentration dynamics in the solid phase.

$$
\begin{gathered}
\frac{\partial c_{s}^{-}}{\partial t}(r, t)=D_{s}^{-}\left[\frac{2}{r} \frac{\partial c_{s}^{-}}{\partial r}(r, t)+\frac{\partial^{2} c_{s}^{-}}{\partial r^{2}}(r, t)\right], \\
\frac{\partial c_{s}^{+}}{\partial t}(r, t)=D_{s}^{+}\left[\frac{2}{r} \frac{\partial c_{s}^{+}}{\partial r}(r, t)+\frac{\partial^{2} c_{s}^{+}}{\partial r^{2}}(r, t)\right], \\
\frac{\partial c_{s}^{-}}{\partial r}(0, t)=0, \quad \frac{\partial c_{s}^{-}}{\partial r}\left(R_{s}^{-}, t\right)=\frac{I(t)}{D_{s}^{-} F a^{-} A L^{-}}, \\
\frac{\partial c_{s}^{+}}{\partial r}(0, t)=0, \quad \frac{\partial c_{s}^{+}}{\partial r}\left(R_{s}^{+}, t\right)=-\frac{I(t)}{D_{s}^{+} F a^{+} A L^{+}} .
\end{gathered}
$$

The Neumann boundary conditions at $r=R_{s}^{+}$and $r=R_{s}^{-}$ signify the flux entering the electrode is proportional to the input current $I(t)$. The Neumann boundary conditions at $r=0$ are spherical symmetry conditions and required for well-posedness. The measured terminal voltage is governed by a combination of electric overpotential, electrode thermodynamics, and Butler-Volmer kinetics. The end result is

$$
\begin{aligned}
V(t)= & \frac{R T}{\alpha F} \sinh ^{-1}\left(\frac{I(t)}{2 a^{+} A L^{+} i_{0}^{+}\left(c_{s s}^{+}(t)\right)}\right) \\
& -\frac{R T}{\alpha F} \sinh ^{-1}\left(\frac{I(t)}{2 a^{-} A L^{-} i_{0}^{-}\left(c_{s s}^{-}(t)\right)}\right) \\
& +U^{+}\left(c_{s s}^{+}(t)\right)-U^{-}\left(c_{s s}^{-}(t)\right)+R_{f} I(t),
\end{aligned}
$$

where the exchange current density $i_{0}^{j}$ and solid-electrolyte surface concentration $c_{s s}^{j}$ are, respectively

$$
\begin{aligned}
i_{0}^{j}\left(c_{s s}^{j}\right) & =k^{j} \sqrt{c_{e}^{0} c_{s s}^{j}(t)\left(c_{s, \max }^{j}-c_{s s}^{j}(t)\right)}, \\
c_{s s}^{j}(t) & =c_{s}^{j}\left(R_{s}^{j}, t\right), \quad \mathrm{j} \in\{+,-\} .
\end{aligned}
$$

Functions $U^{+}(\cdot)$ and $U^{-}(\cdot)$ in (5) are the open circuit potentials of each electrode material, given the surface concentration. Mathematically, these are strictly monotonically decreasing functions. This fact implies the inverse of their derivatives is finite, a property that is required in Section II-C. Further details on the electrochemical principles used to derive these equations can be found in [3], [4]. The SPM parameter definitions can be found in [14].

Note that the bulk anode SOC is defined as the normalized volume sum

$$
\operatorname{SOC}(t)=\frac{3}{c_{s, \max }^{-}\left(R_{s}^{-}\right)^{3}} \int_{0}^{R_{s}^{-}} r^{2} c_{s}^{-}(r, t) d r .
$$

This model contains the property that the total number of lithium ions is conserved [7]. Mathematically, $\frac{d}{d t}\left(n_{L i}\right)=0$, where

$$
\begin{aligned}
n_{L i}= & \frac{\varepsilon_{s}^{+} L^{+} A}{\frac{4}{3} \pi\left(R_{s}^{+}\right)^{3}} \int_{0}^{R_{s}^{+}} 4 \pi r^{2} c_{s}^{+}(r, t) d r \\
& +\frac{\varepsilon_{s}^{-} L^{-} A}{\frac{4}{3} \pi\left(R_{s}^{-}\right)^{3}} \int_{0}^{R_{s}^{-}} 4 \pi r^{2} c_{s}^{-}(r, t) d r .
\end{aligned}
$$

This property is important, as it relates the total concentration of lithium in the cathode and anode. In previous work [8], [14], we have leveraged this fact to perform model reduction for the state estimation problem.

\section{B. Observability \& Model Reduction}

As previously demonstrated [8], [14], the SPM is not completely observable from measurements of voltage and current only. Consequently, the SPM is reduced by approximating the cathode diffusion dynamics (2) by its equilibrium. This renders a completely observable model.

The reduced SPM has a PDE given by (1), boundary conditions given by (3), and output equation

$$
\begin{aligned}
V(t)= & \frac{R T}{\alpha^{+} F} \sinh ^{-1}\left(\frac{I(t)}{2 a^{+} A L^{+} i_{0}^{+}\left(\alpha c_{s s}^{-}(t)+\beta\right)}\right) \\
& -\frac{R T}{\alpha^{-} F} \sinh ^{-1}\left(\frac{I(t)}{2 a^{-} A L^{-} i_{0}^{-}\left(c_{s s}^{-}(t)\right)}\right) \\
& +U^{+}\left(\alpha c_{s s}^{-}(t)+\beta\right)-U^{-}\left(c_{s s}^{-}(t)\right)-R_{f} I(t) .
\end{aligned}
$$

Note that $c_{s s}^{+}(t)$ has been replaced by $\alpha c_{s s}^{-}(t)+\beta$. This is the critical detail of the reduced SPM. The equilibrium of the cathode states (i.e., $c_{s}^{+}(r, t)=c_{s s}^{+}(t)$ ) is computed from the conservation of Li property in (9) to produce the relationship

$$
c_{s S}^{+}(t)=\frac{1}{\varepsilon_{s}^{+} L^{+} A}\left[n_{L i}-\varepsilon_{s}^{-} L^{-} A c_{s s}^{-}(t)\right],
$$

where $\alpha=-\frac{\varepsilon_{s}^{-} L^{-}}{\varepsilon_{s}^{+} L^{+}}$and $\beta=\frac{n_{L i}}{\varepsilon_{s}^{+} L^{+} A}$.

\section{Output Function Inversion}

The reduced SPM contains linear dynamics and a nonlinear output function. In general, an output injection-based estimator would be nonlinear for this class of systems. However, a linear estimator is implemented in this paper by injecting the boundary state error as in [8], [14]. This requires the boundary state to be calculated from the measured voltage as 


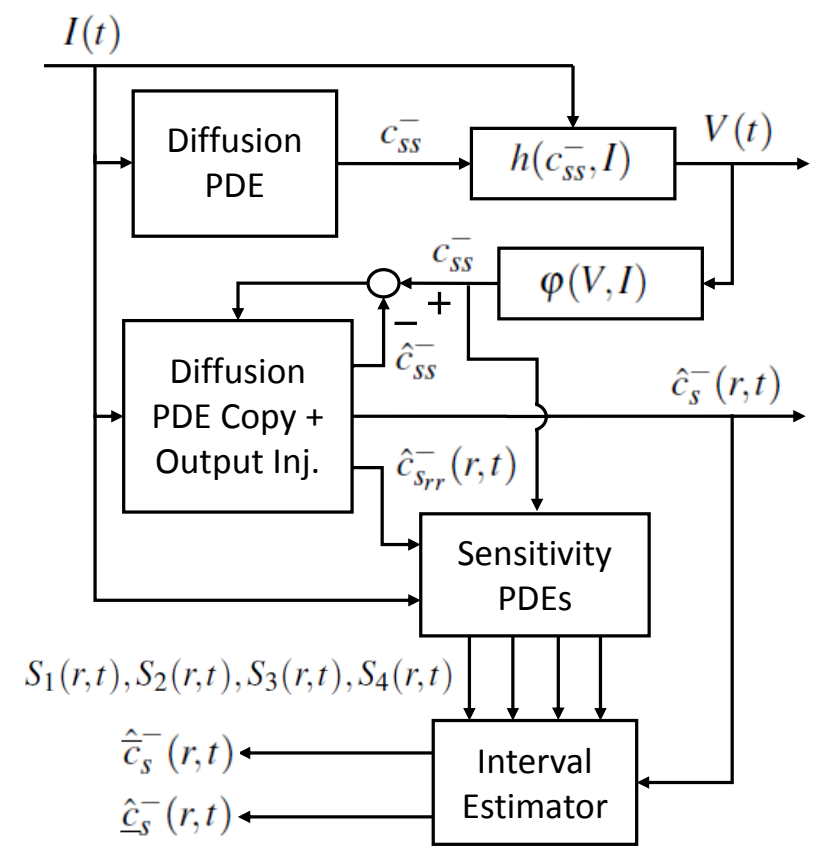

Fig. 2. Block diagram of estimation scheme where the boundary state error is injected into the estimator. The use of the boundary state $c_{s s}^{-}$is determined by $\varphi(V, I)$, which inverts the nonlinear output w.r.t. the state, uniformly in the input current. The double spatial derivative estimates $\hat{c}_{s_{r r}}^{-}(r, t)$ along with input current $I(t)$ and output inversion $\varphi(V, I)$ are fed into the sensitivity PDEs. The sensitivity estimates $S_{1}(r, t), S_{2}(r, t), S_{3}(r, t), S_{4}(r, t)$ along with the concentration estimates $\hat{c}_{s}^{-}$are used to calculate the interval estimates $\hat{c}_{s}^{-}(r, t), \hat{\bar{c}}_{s}^{-}(r, t)$.

shown by the block diagram in Fig. 2. The output function is invertible w.r.t. the boundary state $c_{s s}^{-}$, uniformly in the input current $I(t)$. We show this by defining $h: \mathbb{R} \times \mathbb{R} \rightarrow \mathbb{R}$, such that $V(t)=h\left(c_{s s}^{-}(t), I(t)\right)$. The horizontal line test can be used to show that $h$ is a one to one function w.r.t. $c_{s s}^{-}(t)$, uniformly in $I(t)$. As a result it is possible to determine the inverse function $\varphi$ where $c_{s s}^{-}(t)=\varphi(V(t), I(t))$.

\section{Normalization and State Transformation}

Next we perform normalization and state transformation to simplify the observer structure as done in [8], [14]. First scale the radial $r$ and time $t$ coordinates as follows

$$
\bar{r}=\frac{r}{R_{s}^{-}}, \quad \bar{t}=\frac{D_{s}^{-}}{\left(R_{s}^{-}\right)^{2}} t .
$$

Henceforth we will drop the bars over the space and time coordinates to simplify notation. Next we perform a state transformation to eliminate the first spatial derivative in the spherical diffusion equation (1). Namely, let

$$
c(r, t)=r c_{s}^{-}(r, t) .
$$

This normalization and state transformation produces the following PDE with Dirichlet and Robin boundary conditions

$$
\begin{aligned}
\frac{\partial c}{\partial t}(r, t) & =\varepsilon \frac{\partial^{2} c}{\partial r^{2}}(r, t), \\
c(0, t) & =0, \\
\frac{\partial c}{\partial r}(1, t)-c(1, t) & =-q \rho I(t) .
\end{aligned}
$$

and nonlinear output map given by (10) where $c_{s s}^{+}=$ $\alpha c(1, t)+\beta$ (see (11)), and $c_{s s}^{-}=c(1, t)$. The parameter $\rho=R_{s}^{-} /\left(D_{s}^{-} F a^{-} A L^{-}\right)$groups parameters together. The parameters $\varepsilon$ and $q$ are nominally equal to one. Respectively, they represent uncertainty in the diffusion and boundary input coefficients. In the following sections, we derive an interval observer that maps uncertainty in these parameters to bounds on the state estimates.

\section{BACKSTEPPING PDE OBSERVER DESIGN}

The sensitivity-based interval PDE observer is based upon the backstepping design reported in [8], [14]. We summarize the observer design here. The state estimator structure consists of a copy of the plant (14)-(16) plus boundary state error injection, as follows

$$
\begin{aligned}
\frac{\partial \hat{c}}{\partial t}(r, t) & =\varepsilon \frac{\partial^{2} \hat{c}}{\partial r^{2}}(r, t)+p_{1}(r) \tilde{c}(1, t), \\
\hat{c}(0, t) & =0, \\
\frac{\partial \hat{c}}{\partial r}(1, t)-\hat{c}(1, t) & =-q \rho I(t)+p_{10} \tilde{c}(1, t),
\end{aligned}
$$

where the boundary state error is given by

$$
\tilde{c}(1, t)=\gamma \varphi(V(t), I(t))-\hat{c}(1, t) .
$$

Function $\varphi(V(t), I(t))$ generates the boundary state from measured voltage and current by inverting the output function, as demonstrated visually by the block diagram in Fig. 2, as explained in Section II-C. The parameter $\gamma$ represents the uncertainty in the output inversion, and is nominally equal to one. The backstepping approach [13] is applied to design the output injection gains $p_{1}(r)$ and $p_{10}$, resulting in

$$
\begin{aligned}
p_{1}(r) & =\frac{-\lambda r}{2 x}\left[I_{1}(x)-\frac{2 \lambda}{x} I_{2}(x)\right], \text { where } x=\sqrt{\lambda\left(r^{2}-1\right)} \\
p_{10} & =\frac{3-\lambda}{x}
\end{aligned}
$$

and $I_{1}(x)$ and $I_{2}(x)$ are, respectively, the first and second order modified Bessel functions of the first kind.

\section{Observer Sensitivity EquATIONS}

The main contribution of this paper is an interval observer based upon the aforementioned backstepping observer, and the following sensitivity equations. Consider the parameterized PDE backstepping SPM observer

$$
\begin{aligned}
\frac{\partial \hat{c}}{\partial t}(r, t)=\varepsilon \frac{\partial^{2} \hat{c}}{\partial r^{2}}(r, t) & +p_{1}(r)[\gamma \varphi(V(t), I(t))-\hat{c}(1, t)], \\
\hat{c}\left(r, t_{0}\right)= & \delta \hat{c}_{0}(r) \\
\hat{c}(0, t)= & 0 \\
\frac{\partial \hat{c}}{\partial r}(1, t)-\hat{c}(1, t)= & -q \rho I(t) \\
& +p_{10}[\gamma \varphi(V(t), I(t))-\hat{c}(1, t)]
\end{aligned}
$$

where $\theta=[\varepsilon, q, \gamma, \delta]^{T}$ represents the uncertain parameters, whose values are nominally given by $\theta_{0}=\left[\varepsilon_{0}, q_{0}, \gamma_{0}, \delta_{0}\right]^{T}$ $=[1,1,1,1]^{T}$. The parameter $\delta$ represents uncertainty in 
the initial condition of the observer. Suppose the nominal observer has a unique solution denoted $\hat{c}\left(r, t ; \theta_{0}\right)$. We seek to study variations of this solution due to variations in $\theta$.

In the following, we derive sensitivity equations w.r.t. $\varepsilon$ using the procedure outlined by Khalil in Chapter 3 of [12]. The remaining sensitivity equations w.r.t. $q, \gamma$, and $\delta$ follow an identical process. Let us re-write the PDE (23) in partial integro-differential equation (PIDE) form

$$
\begin{aligned}
\hat{c}(r, t)=\delta \hat{c}_{0}(r)+\int_{t_{0}}^{t}\left[\varepsilon \hat{c}_{r r}(r, s ; \theta)\right. & \\
+ & \left.p_{1}(r)(\gamma \varphi(V(t), I(t))-\hat{c}(1, s ; \theta))\right] d s, \\
\hat{c}\left(r, t_{0}\right)= & \delta \hat{c}_{0}(r), \\
\hat{c}(0, t)= & 0, \\
\hat{c}_{r}(1, t)-\hat{c}(1, t)= & -q \rho I(t) \\
& +p_{10}[\gamma \varphi(V(t), I(t))-\hat{c}(1, t)],
\end{aligned}
$$

where $\hat{c}_{r r}=\partial^{2} \hat{c} / \partial r^{2}$. Taking partial derivatives of both sides w.r.t. $\varepsilon$ yields

$$
\begin{gathered}
\frac{\partial \hat{c}}{\partial \varepsilon}(r, t)=\int_{t_{0}}^{t}\left[\varepsilon \frac{\partial \hat{c}_{r r}}{\partial \varepsilon}(r, s ; \theta)+\hat{c}_{r r}(r, s ; \theta)-p_{1}(r) \frac{\partial \hat{c}}{\partial \varepsilon}(1, s ; \theta)\right] d s \\
\frac{\partial \hat{c}}{\partial \varepsilon}\left(r, t_{0}\right)=\frac{\partial \hat{c}}{\partial \varepsilon}(0, t)=0 \\
\frac{\partial \hat{c}_{r}}{\partial \varepsilon}(1, t)-\frac{\partial \hat{c}}{\partial \varepsilon}(1, t)=-p_{10} \frac{\partial \hat{c}}{\partial \varepsilon}
\end{gathered}
$$

where $\hat{c}_{r}=\partial \hat{c} / \partial r$, and since $c_{0}(r)$ is independent of $\varepsilon$. We denote $\hat{c}_{\varepsilon}=\partial \hat{c} / \partial \varepsilon$, and change the order of differentiation in the first term on the RHS of (31)

$$
\begin{aligned}
\hat{c}_{\varepsilon}(r, t)=\int_{t_{0}}^{t}\left[\varepsilon \frac{\partial^{2} \hat{c}_{\varepsilon}}{\partial r^{2}}(r, s ; \theta)+\hat{c}_{r r}(r, s ; \theta)-p_{1}(r) \hat{c}_{\varepsilon}(1, s ; \theta)\right] d s \\
\hat{c}_{\varepsilon}\left(r, t_{0}\right)=\hat{c}_{\varepsilon}(0, t)=0 \\
\frac{\partial \hat{c}_{\varepsilon}}{\partial r}(1, t)-\hat{c}_{\varepsilon}(1, t)=-p_{10} \hat{c}_{\varepsilon}(1, t) .
\end{aligned}
$$

Differentiating w.r.t. time, we find that $\hat{c}_{\varepsilon}(r, t)$ verifies the PDE

$$
\begin{array}{r}
\frac{\partial}{\partial t} c_{\varepsilon}(r, t)=\varepsilon \frac{\partial^{2}}{\partial r^{2}} \hat{c}_{\varepsilon}(r, t ; \theta)+\hat{c}_{r r}(r, t ; \theta)-p_{1}(r) \hat{c}_{\varepsilon}(1, t ; \theta) \\
\hat{c}_{\varepsilon}\left(r, t_{0}\right)=\hat{c}_{\varepsilon}(0, t)=0 \\
\frac{\partial \hat{c}_{\varepsilon}}{\partial r}(1, t)-\hat{c}_{\varepsilon}(1, t)=-p_{10} \hat{c}_{\varepsilon}(1, t)
\end{array}
$$

When $\theta=\theta_{0}$, then the RHS of (37) depends only on the nominal solution $\hat{c}\left(r, t ; \theta_{0}\right)$. Let $S_{1}(r, t)=\hat{c}_{\varepsilon}\left(r, t ; \theta_{0}\right)$ be the sensitivity function. Then $S_{1}(r, t)$ is the solution of the "Sensitivity PDE":

$$
\begin{aligned}
S_{1_{t}}(r, t)=\varepsilon_{0} S_{1_{r r}}\left(r, t ; \theta_{0}\right)+\hat{c}_{r r}\left(r, t ; \theta_{0}\right)-p_{1}(r) S_{1}\left(1, t ; \theta_{0}\right) & \\
S_{1}\left(r, t_{0}\right) & =S_{1}(0, t)=0 \\
S_{1_{r}}(1, t)-S_{1}(1, t) & =-p_{10} S_{1}(1, t)
\end{aligned}
$$

Note that the sensitivity PDE is linear in $S_{1}(r, t)$ and driven by exogenous signal $\hat{c}_{r r}\left(r, t ; \theta_{0}\right)$.

Similarly, the sensitivity equations w.r.t. $q$ are computed as

$$
\begin{gathered}
S_{2_{t}}(r, t)=\varepsilon_{0} S_{2_{r r}}\left(r, t ; \theta_{0}\right)-p_{1}(r) S_{2}(1, t), \\
S_{2}\left(r, t_{0}\right)=S_{2}(0, t)=0, \\
S_{2_{r}}(1, t)-S_{2}(1, t)=-\rho I(t)-p_{10} S_{2}(1, t),
\end{gathered}
$$

where $S_{2}(r, t)=\hat{c}_{q}\left(r, t ; \theta_{0}\right)$. The sensitivity PDE is linear in $S_{2}(r, t)$ and driven by exogenous signal $I(t)$. The sensitivity equations w.r.t. $\gamma$ are computed as

$$
\begin{gathered}
S_{3_{t}}(r, t)=\varepsilon_{0} S_{3_{r r}}\left(r, t ; \theta_{0}\right)+p_{1}(r) \varphi(V(t), I(t))-p_{1}(r) S_{3}(1, t), \\
S_{3}\left(r, t_{0}\right)=S_{3}(0, t)=0, \\
S_{3_{r}}(1, t)-S_{3}(1, t)=p_{10} \varphi(V(t), I(t))-p_{10} S_{3}(1, t),
\end{gathered}
$$

where $S_{3}(r, t)=\hat{c}_{\gamma}\left(r, t ; \theta_{0}\right)$. The sensitivity PDE is linear in $S_{3}(r, t)$ and driven by exogenous signal $\varphi(V(t), I(t))$. Lastly, the sensitivity equations w.r.t. $\delta$ are computed as

$$
\begin{gathered}
S_{4_{t}}(r, t)=\varepsilon_{0} S_{4_{r r}}\left(r, t ; \theta_{0}\right)-p_{1}(r) S_{4}(1, t), \\
S_{4}\left(r, t_{0}\right)=\hat{c_{0}}(r) \\
S_{4}(0, t)=0 \\
S_{4_{r}}(1, t)-S_{4}(1, t)=-p_{10} S_{4}(1, t)
\end{gathered}
$$

where $S_{4}(r, t)=\hat{c}_{\delta}\left(r, t ; \theta_{0}\right)$. The sensitivity PDE is linear in $S_{4}(r, t)$, and is autonomous. Note that $S_{1}(r, t), S_{2}(r, t), S_{3}(r, t), S_{4}(r, t)$ quantify the sensitivity of the estimated states to variations in the uncertain parameter values. We exploit this property to also perform a sensitivity analysis in Section VI.

Consequently, when $\theta$ is close to the nominal value $\theta_{0}$, we can approximate the solution $\hat{c}(r, t ; \theta)$ around the nominal solution $\hat{c}\left(r, t ; \theta_{0}\right)$ to first-order accuracy by,

$$
\begin{aligned}
\hat{c}(r, t ; \theta):= & \hat{c}\left(r, t ; \theta_{0}\right)+S_{1}(r, t)\left(\varepsilon-\varepsilon_{0}\right)+S_{2}(r, t)\left(q-q_{0}\right) \\
& +S_{3}(r, t)\left(\gamma-\gamma_{0}\right)+S_{4}(r, t)\left(\delta-\delta_{0}\right) .
\end{aligned}
$$

\section{Sensitivity-BAsed Interval Observer}

We are now positioned to formulate an interval observer based upon the sensitivity equations derived in the previous section. First, we assume the parameters $\theta=[\varepsilon, q, \gamma, \delta]^{T}$ are unknown but bounded as follows

$$
\underline{\varepsilon} \leq \varepsilon \leq \bar{\varepsilon}, \quad \underline{q} \leq q \leq \bar{q}, \quad \underline{\gamma} \leq \gamma \leq \bar{\gamma}, \quad \underline{\delta} \leq \delta \leq \bar{\delta},
$$

where the bounds $\underline{\varepsilon}, \underline{q}, \underline{\gamma}, \underline{\delta}, \bar{\varepsilon}, \bar{q}, \bar{\gamma}, \bar{\delta}$ are known. Then the nominal state estimate $\hat{c}(r, t)$ is generated from the PDE backstepping observer with interval estimates $\underline{\hat{c}}(r, t), \hat{\bar{c}}(r, t)$ computed as

$$
\begin{array}{r}
\hat{\underline{c}}(r, t)=\min \left\{\hat{c}(r, t) \pm S_{1}(r, t)\left(\underline{\varepsilon}-\varepsilon_{0}\right) \pm S_{2}(r, t)\left(\underline{q}-q_{0}\right)\right. \\
\left. \pm S_{3}(r, t)\left(\underline{\gamma}-\gamma_{0}\right) \pm S_{4}(r, t)\left(\underline{\delta}-\delta_{0}\right)\right\}, \\
\hat{\bar{c}}(r, t)=\max \left\{\hat{c}(r, t) \pm S_{1}(r, t)\left(\bar{\varepsilon}-\varepsilon_{0}\right) \pm S_{2}(r, t)\left(\bar{q}-q_{0}\right)\right. \\
\left. \pm S_{3}(r, t)\left(\bar{\gamma}-\gamma_{0}\right) \pm S_{4}(r, t)\left(\bar{\delta}-\delta_{0}\right)\right\},
\end{array}
$$



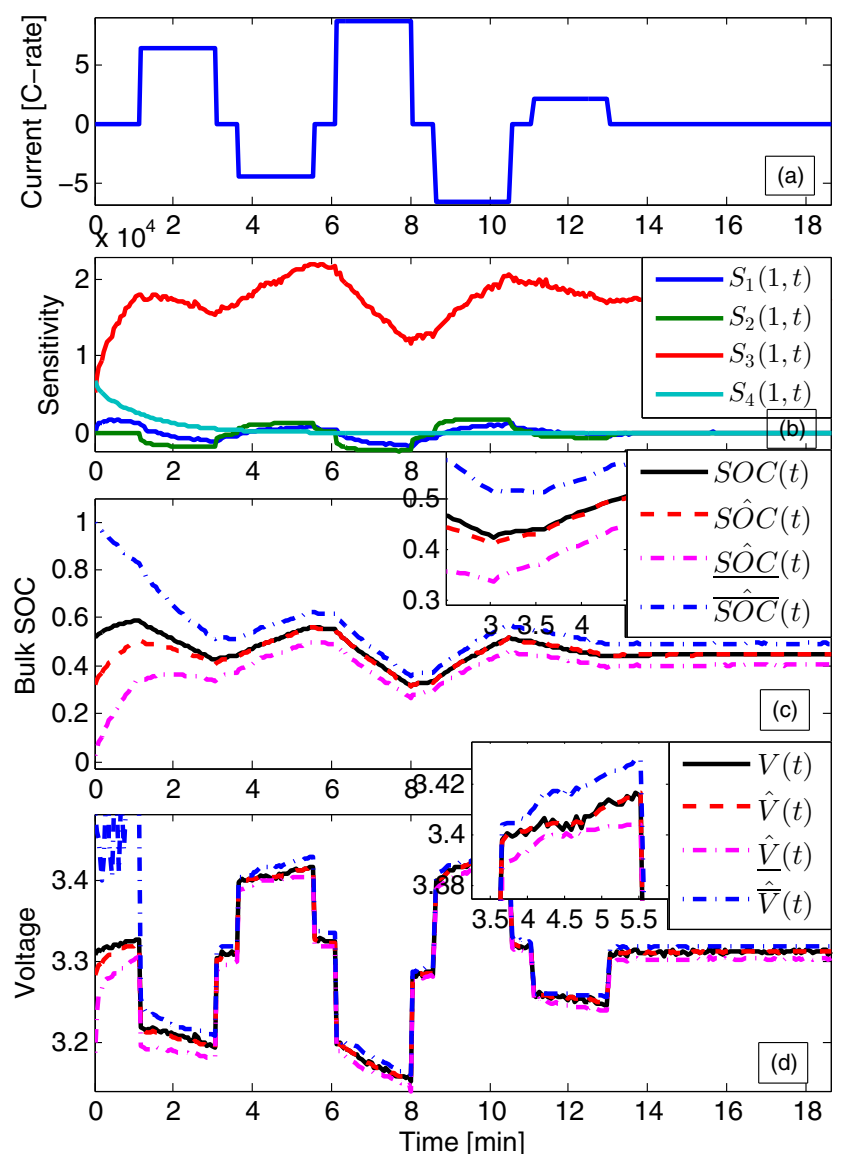

Fig. 3. Pulse Charge/Discharge Cycle (a) Input current. (b) Sensitivity. (c) Bulk SOC. (d) Output Voltage.

where $S_{1}(r, t), S_{2}(r, t), S_{3}(r, t), S_{4}(r, t)$ are solutions to the sensitivity PDEs (40)-(52). These interval estimates consider all possible cases where $S_{1}(r, t), S_{2}(r, t), S_{3}(r, t), S_{4}(r, t)$ are positive or negative. This allows for the absolute $\min / \max$ to be used as the interval estimates, which are then used to find the interval estimates of bulk SOC $(\underline{\operatorname{SOC} C}(t), \overline{\operatorname{SOC}}(t))$ and voltage $(\underline{\hat{V}}(t), \hat{\bar{V}}(t))$.

\section{Simulations}

In this section we present simulation examples of the interval PDE observer for various charge/discharge cycles, along with a sensitivity analysis. We apply the observer to the reduced SPM, apply the sensitivity equations to the observer, whose results are then used in the interval observer. We work in the normalized $(r, t)$ coordinates but retain the original state realization. The model parameters originate from [9] for a commercial $\mathrm{LiFePO}_{4}$ cell. Estimation gain parameter $\lambda=-5$ from (21)-(22), as in [14]. The uncertain parameters for the interval observer are set at $\underline{\theta}=[0.9,0.9,0.9,0.0]^{T}$ and $\bar{\theta}=[1.1,1.1,1.1,3.3]^{T}$ which represents a $\pm 10 \%$ deviation from the nominal parameters $\varepsilon_{0}, q_{0}, \gamma_{0}$ and the range of physically meaningful observer initial conditions for the nominal parameter $\delta_{0}$. All PDE models are implemented

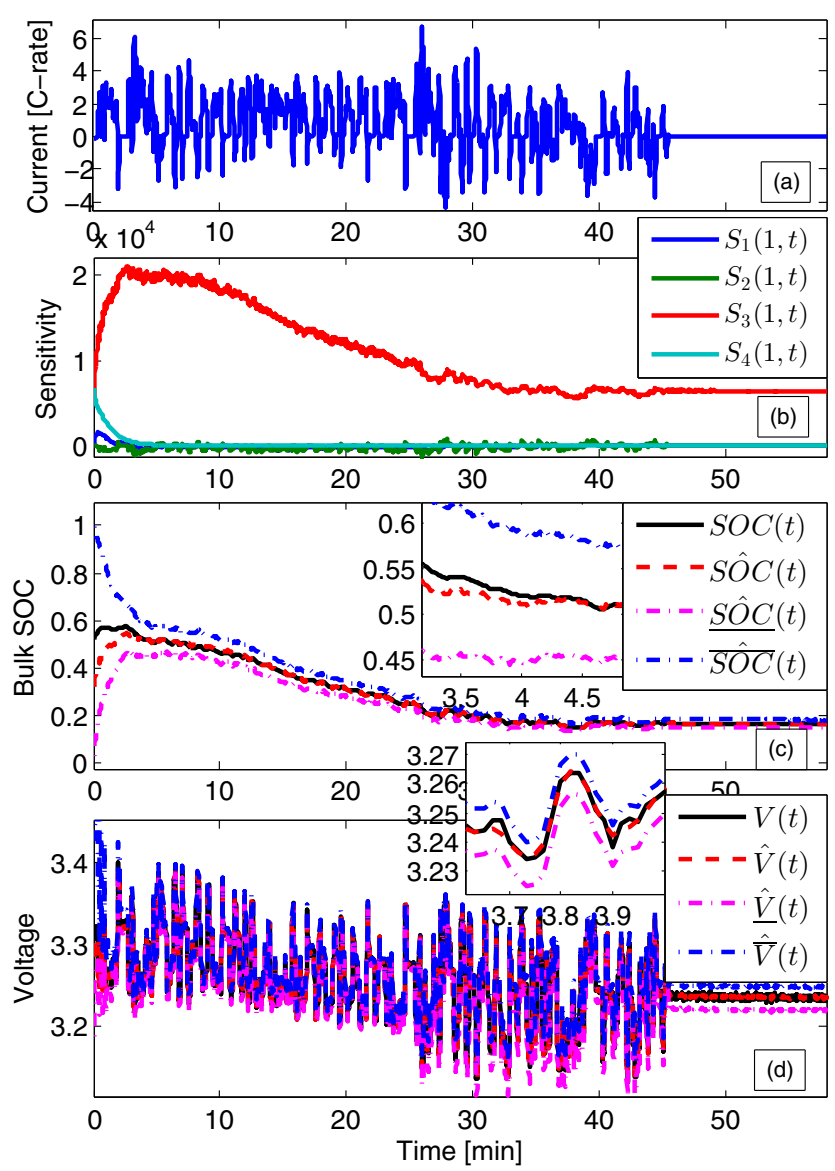

Fig. 4. UDDSx2 Charge/Discharge Cycle (a) Input current. (b) Sensitivity. (c) Bulk SOC. (d) Output Voltage.

using the finite central difference method. The SPM plant and observer states are initialized at different values to demonstrate uncertainty in initial conditions. Zero mean normally distributed noise with a standard deviation of $2 \mathrm{mV}$ is added to the voltage measurement.

First, a pulse current charge/discharge cycle is applied. Figure 3(a) shows the applied current in terms of C-rate (normalized current against charge capacity), where positive values indicate discharge rates. Figure 3(b) shows the resulting sensitivities, where $S_{3}$ is largest, followed by $S_{2}, S_{1}$, and $S_{4}$. This indicates that the observer system is most sensitive to perturbations in $\gamma$, followed by $q$, then $\varepsilon$, and finally $\delta$. More specifically, the observer system is most sensitive to the output inversion $\varphi$ as $\gamma$ represents its uncertainty. Figure 3(c) shows the true and estimated bulk SOC along with the upper and lower interval estimates. We see that the estimate converges to within $1 \%$ of the the true value at $\mathrm{t}=4.13 \mathrm{~min}$. We also see that the interval estimates encapsulate the true SOC within reasonable bounds. Figure 3(d) shows the plant output voltage with noise and estimated voltage along with the upper and lower interval estimates. At $\mathrm{t}=1.19 \mathrm{~min}$ the voltage error between the estimated and true voltage with noise is less than $3 \mathrm{mV}$. We also see that the interval estimates encapsulate the measured voltage within reasonable bounds. 


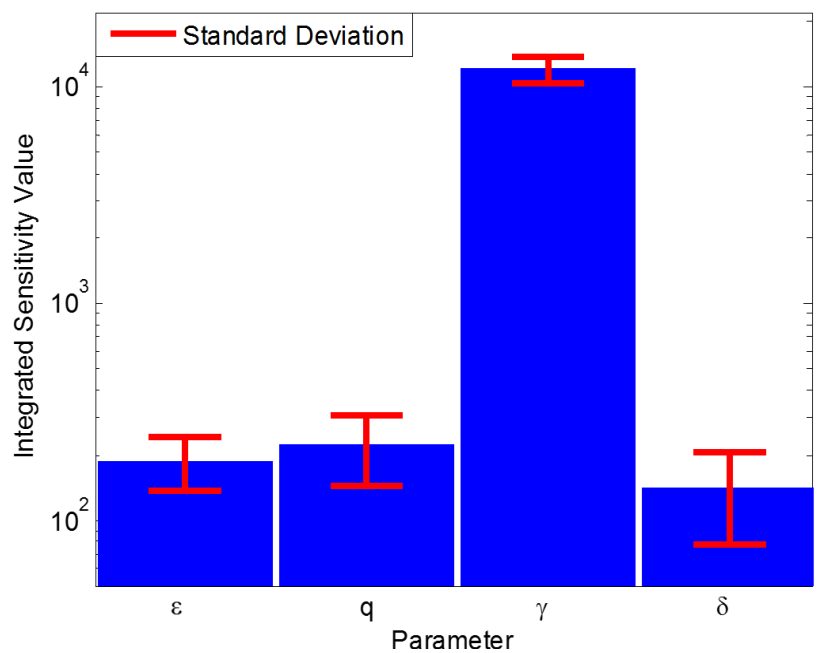

Fig. 5. Normalized parameter sensitivity ranking (average in blue, standard deviation in red) across various electric vehicle-like charge/discharge cycles (UDDSx2, US06x3, SC04x4, LA92x2, DC1, DC2).

Next, an electric vehicle-like charge/discharge cycle consisting of two concatenated urban dynamometer driving schedules (UDDS) is applied. Figure 4(a) shows the applied current in terms of C-rate. Figure 4(b) shows the resulting sensitivities, where $S_{3}$ is largest, followed by $S_{2}, S_{1}$, and $S_{4}$. Note that this is the same observation as seen with the pulse current profile. Figure 4(c) shows the true and estimated bulk SOC along with the upper and lower interval estimates. We see that the estimate converges to within $1 \%$ of the the true value at $\mathrm{t}=4.25 \mathrm{~min}$. We also see that the interval estimates encapsulate the true SOC within reasonable bounds. Figure 4(d) shows the plant output voltage with noise and estimated voltage along with the upper and lower interval estimates. At $\mathrm{t}=1.17 \mathrm{~min}$ the voltage error between the estimated and true voltage with noise is less than $3 \mathrm{mV}$. We also see that the interval estimates encapsulate the true voltage with noise within reasonable bounds.

Lastly, a set of electric vehicle-like charge/discharge cycles (US06x3, SC04x4, LA92x2, DC1, DC2) [16] are applied to further understand the effects of parameter uncertainty through sensitivity analysis on the observer system. We rank the parameters based on the integrated absolute value of each sensitivity normalized by time,

$$
S_{\text {rank }_{i}}=\frac{1}{T} \int_{0}^{T}\left|S_{i}(s)\right| d s,
$$

where $i \in[1,2,3,4]$, and $T$ is the total time. Figure 5 shows that estimated SOC is most sensitive to perturbations in $\gamma$, followed by $q, \varepsilon$, and finally $\delta$. Consequently, accurate output inversion is most important for accurate SOC estimation.

\section{CONCLUSIONS}

This paper examines the sensitivity of the previously developed backstepping PDE estimator [8], [14] due to perturbations in the nominal uncertain parameters. It then combines the resulting sensitivities with the observer estimates to create upper and lower interval estimates for a given deviation in the nominal uncertain parameters, and the range of physically meaningful initial conditions. The sensitivity results are also used to rank parameter sensitivity to understand which parameters have the greatest effect on the observer estimates. This is important for prioritizing which parameters to identify in a system identification process.

\section{REFERENCES}

[1] T. Ahonen, Tomi Ahonen Almanac 2012- Mobile Telecoms Industry Review. Lulu.com, 2012.

[2] Electric Drive Transportation Association. (2012) Electric drive vehicle sales figures (U.S. Market) - EV sales. [Online]. Available: http://electricdrive.org/index.php?ht=d/sp/i/20952/pid/20952

[3] N. A. Chaturvedi, R. Klein, J. Christensen, J. Ahmed, and A. Kojic, "Algorithms for advanced battery-management systems," IEEE Control Systems Magazine, vol. 30, no. 3, pp. 49 - 68, 2010.

[4] K. Thomas, J. Newman, and R. Darling, Advances in Lithium-Ion Batteries. New York, NY USA: Kluwer Academic/Plenum Publishers, 2002, ch. 12: Mathematical modeling of lithium batteries, pp. 345392.

[5] K. A. Smith, C. D. Rahn, and C.-Y. Wang, "Model-based electrochemical estimation and constraint management for pulse operation of lithium ion batteries," IEEE Transactions on Control Systems Technology, vol. 18, no. 3, pp. $654-663,2010$.

[6] D. D. Domenico, A. Stefanopoulou, and G. Fiengo, "Lithium-Ion Battery State of Charge and Critical Surface Charge Estimation Using an Electrochemical Model-Based Extended Kalman Filter," Journal of Dynamic Systems, Measurement, and Control, vol. 132, no. 6, p. 061302, 2010.

[7] R. Klein, N. A. Chaturvedi, J. Christensen, J. Ahmed, R. Findeisen, and A. Kojic, "Electrochemical Model Based Observer Design for a Lithium-Ion Battery," IEEE Transactions on Control Systems Technology, vol. 21, no. 2, pp. 289-301, March 2013.

[8] S. J. Moura, N. Chaturvedi, and M. Krstic, "Adaptive PDE Observer for Battery SOC/SOH Estimation via an Electrochemical Model," ASME Journal of Dynamic Systems, Measurement, and Control, vol. 136, no. 1, pp. 011015-011026, Oct 2013.

[9] J. C. Forman, S. J. Moura, J. L. Stein, and H. K. Fathy, "Genetic identification and Fisher identifiability analysis of the Doyle-FullerNewman model from experimental cycling of a LiFePO4 cells," Journal of Power, vol. 210, pp. 263-275, 2012.

[10] C. Combastel, "A state bounding observer for uncertain non-linear continuous-time systems based on zonotopes," in Decision and Control, 2005 and 2005 European Control Conference. CDC-ECC '05. 44th IEEE Conference on, Dec 2005, pp. 7228-7234.

[11] T. Raissi, D. Efimov, and A. Zolghadri, "Interval state estimation for a class of nonlinear systems," Automatic Control, IEEE Transactions on, vol. 57, no. 1, pp. 260-265, Jan 2012.

[12] H. K. Khalil, Nonlinear Systems, 3rd ed. Prentice Hall, 2002.

[13] M. Krstic and A. Smyshlyaev, Boundary Control of PDEs: A Course on Backstepping Designs. Philadelphia, PA: Society for Industrial and Applied Mathematics, 2008.

[14] S. J. Moura, N. Chaturvedi, and M. Krstic, "PDE Estimation Techniques for Advanced Battery Management Systems - Part I: SOC Estimation," in Proceedings of the 2012 American Control Conference, Montreal, Canada, June 2012.

[15] S. Santhanagopalan and R. E. White, "Online estimation of the state of charge of a lithium ion cell," Journal of Power Sources, vol. 161, no. 2, pp. 1346 - 1355, 2006.

[16] S. Moura, J. Stein, and H. Fathy, "Battery-Health Conscious Power Management in Plug-In Hybrid Electric Vehicles via Electrochemical Modeling and Stochastic Control," Control Systems Technology, IEEE Transactions on, vol. 21, no. 3, pp. 679-694, 2013. 